\title{
Integral Equation Method for Inverse Scattering Problem from the Far-Field Data
}

\author{
Yuqing $\mathrm{Hu}^{*}$ \\ School of Science, Jinling Institute of Technology, Nanjing, Jiangsu 211169, China
}

Received 14 February 2020; Accepted (in revised version) 25 October 2020

\begin{abstract}
Consider the inverse scattering problem in terms of Helmholtz equation. We study a simply connected domain with oblique derivative boundary condition. In the case of constant $\lambda$, given a finite number of incident wave, the shape of the scatterer is reconstructed from the measured far-field data. We propose a Newton method which is based on the nonlinear boundary integral equation. After computing the Fréchet derivatives with respect to the unknown boundary, the nonlinear equation is transformed to its linear form, then we show the iteration scheme for the inverse problem. We conclude our paper by presenting several numerical examples for shape reconstruction to show the validity of the method we presented.
\end{abstract}

AMS subject classifications: 35R30, 65F22, 65R20, 65R32

Key words: Helmholtz equation, oblique derivative problem, nonlinear integral equation, iterative solution, numerics.

\section{Introduction}

The theory of wave field scattering plays a very important role in the field of mathematical physics. For example, the research of electromagnetic wave and acoustic wave scattering has a wide application prospect in radar remote control, space remote sensing, nondestructive testing, target stealth, medical imaging and other technologies. In a broad sense, the theory of scattering studies the relationship between medium and scattering wave. Specifically, if the total field is regarded as the sum of incident field and scattering field, the forward scattering problem is to determine the far-field mode of scattering field or scattering wave by the information and differential equation of incident field. Its mathematical essence is to solve the problem of definite solution of partial differential equation in unbounded domain. The inverse scattering problem of wave field is an important kind of inverse problem. It uses the measurement information outside the medium to detect the properties of the medium, which can not be measured directly.

*Corresponding author.

Email: yqhu@jit.edu.cn (Y. Hu) 
A lot of work has been done on the scattering problem of Dirichlet and Neumann boundary conditions. For the existence and uniqueness of the solution to the direct problem, see $[8,9,12]$. The authors used boundary integral equation method in $[8,12]$ (and references therein). In $[1,9,11,20]$ the inverse problem with Dirichlet and Neumann bounday conditions were considered. The diffraction of tidal waves by an island and barriers on water of constant finite depth are modelized by the two dimensional Helmholtz equation in the free space with oblique derivative boundary conditions. The research of the problem with oblique derivative boundary is still relatively few, mainly because the boundary equation contains the tangential derivative term, which makes it difficult to deal with, but the model is suitable for any wave dynamic description, it is necessary to study it. For the scattering problem with oblique derivative boundary condition, numerical methods were given by the single-layer potential and the angular potential to the positive problem of the open curve in [14-16]. In [18], the author proved the uniqueness of the solution, then used polynomial to approximate the density function and gave the method for solving the solution, but no specific numerical examples were given. In [24], the authors considered the exterior boundary value problem for the two-dimensional Helmholtz equation with generalized oblique derivative boundary condition. They established the linear sampling method (LSM) for reconstructing the boundary of the obstacle from the far-field data.

There are many numerical methods for reconstructing the shape of scatterers, such as linear sampling method, detection method, factor decomposition method, etc. Since the inverse scattering problem is a typical nonlinear ill-posed problem, Newton method and integral equation method are often used to solve it. Newton method is to use Fréchet derivatives to transform the nonlinear equation into its linear form, then use Tikhonov regularization method to deal with the ill-posed problem, see $[2,4,6,7,13,17,19,22]$. In $[6,7]$ the authors applied Fréchet derivatives in Newton method to reconstruct the impedance boundary shape of the corrosion problems. In $[4,22]$ the authors used full linearization and just linearized one of the equations to solve the inverse problems. The integral equation method has been used widely in $[3,5]$.

This paper is devoted to the numerical study of the oblique inverse scattering problem with Helmholtz equation by Newton method. More explicitly, we note that the inverse problem can be reformulated as an operator equation with respect to the boundary of the obstacle. By linearizing this operator equation, we solve the inverse problem by Newton method. First, we give an initial boundary and compute the corresponding far-field pattern. Then, after considering Fréchet differentiability of the far-field operator, we calculate the Fréchet derivatives with respect to the boundary. Finally, using regularization technique, we solve the linearized operator equation to update the boundary. Several numerical examples are presented to show the feasibility of the reconstruction scheme.

The rest of this paper is organized as follows. In Section 2, we show the inverse problem with oblique derivative boundary. Then the inverse problem of reconstructing the shape is transformed into solving the nonlinear boundary integral equation. In Section 3, we consider Fréchet differentiability of the far-field operator as the theoretical foundation 
of Newton method. In Section 4, Newton method is proposed to reconstruct the shape of the boundary. Firstly, the Fréchet derivatives of the unknown boundary is obtained, and the original nonlinear integral equation is transformed into linear integral equation. In addition, the regularized Newton method is used to solve the linear equation which is equivalent to the inverse problem. We end the paper with various numerical examples of reconstructing the boundary shape, showing the validity and efficiency of our method.

\section{Integral equation}

Assume that sound wave propagates in isotropic homogeneous medium. Outside the scatterer, the total field is the superposition of incident wave and scattered wave. Suppose that the incident wave is time harmonic, let the scatterer $D \subset \mathbb{R}^{2}$ be a bounded and impenetrable domain with $\mathcal{C}^{2}$-smooth boundary $\partial D$. The external medium of $D$ is homogeneous and isotropic. Denote by $u^{i}(x)=e^{i k x \cdot d}$ the incident wave with wavenumber $k>0$ and with a unit vector $d, u^{s}$ the corresponding scattered wave, then the total field $u=u^{i}+u^{s}$ satisfies Helmholtz equation out of $D$, i.e.,

$$
\Delta u+k^{2} u=0, \quad x \in \mathbb{R}^{2} \backslash \bar{D} .
$$

In this paper, consider the scattering problem of ocean wave. Under some assumptions, the scattering problem can be summarized as the boundary value problem of Helmholtz equation on the horizontal plane. Suppose that the earth rotation is considered, the total field satisfies the boundary condition with oblique derivative

$$
\frac{\partial u}{\partial v}(x)+i \lambda \frac{\partial u}{\partial \tau}(x)=0, \quad x \in \partial D
$$

where $v$ is the unit outward normal to $\partial D, \tau$ is the unit tangent vector of $\partial D$, obtained by turning $v$ counter clockwise for $\pi / 2$, and the impedance coefficient $|\lambda|<1$ [18]. Specially, $\lambda=0$ without the earth's rotation. Helmholtz equation (2.1) and (2.2) consist of a boundary value problem. In order to ensure the uniqueness of the boundary value problem, for the scattered wave $u^{s}$, we demand that the Sommerfeld radiation condition

$$
\lim _{r \rightarrow \infty} \sqrt{r}\left(\frac{\partial u^{s}}{\partial r}-i k u^{s}\right)=0, \quad r=|x|,
$$

holds uniformly in all directions $\hat{x}=x /|x|$ and $\hat{x} \in \Omega$, where $\Omega$ is the unit circle in $\mathbb{R}^{2}$. In particular, we are interested in the computation of the far-field pattern $u_{\infty}$ of the scattered wave. The far-field pattern describes the behaviour of the scattered wave at infinity

$$
u(x)=\frac{e^{i k|x|}}{\sqrt{|x|}}\left\{u_{\infty}(\hat{x})+\mathcal{O}\left(\frac{1}{|x|}\right)\right\}, \quad|x| \rightarrow \infty,
$$

uniformly for all directions $\hat{x}=x /|x|$. 
Thus, the inverse problem is: reconstruct the shape of the scatterer from the measured far-field data based on (2.1)-(2.3).

From the oblique derivative problem (2.1)-(2.3), we know the scattered wave $u^{s}$ satisfies

$$
\begin{cases}\Delta u^{s}+k^{2} u^{s}=0, & x \in \mathbb{R}^{2} \backslash \bar{D}, \\ \frac{\partial u^{s}}{\partial v}+i \lambda \frac{\partial u^{s}}{\partial \tau}=f, & x \in \partial D, \\ \lim _{r \rightarrow \infty} \sqrt{r}\left(\frac{\partial u^{s}}{\partial r}-i k u^{s}\right)=0, & r=|x|,\end{cases}
$$

where the function $f$ can be written as

$$
f=-\left(\frac{\partial u^{i}}{\partial v}+i \lambda \frac{\partial u^{i}}{\partial \tau}\right)
$$

for the given incident wave $u^{i}$, with $|\lambda|<1[18]$.

Assume $\lambda$ is a nonzero constant, by the jump relation of single layer potential and the boundary condition, we can come to a conclusion that if $-k^{2}$ is not a Dirichlet eigenvalue of the Laplacian in $D$ and $|\lambda|<1$, then the system (2.5) has a unique solution [25].

The uniqueness of this inverse scattering problem has been established in [23]. This section is devoted to the mathematical justification of the integral equation method in our setting.

Define the following boundary integral operators

$$
\begin{array}{ll}
S[\varphi](x):=2 \int_{\partial D} \Phi(x, y) \varphi(y) d s(y), & x \in \partial D, \\
K^{\prime}[\varphi](x):=2 \int_{\partial D} \frac{\partial \Phi(x, y)}{\partial v(x)} \varphi(y) d s(y), \quad x \in \partial D, \\
H^{\prime}[\varphi](x):=2 \int_{\partial D} \frac{\partial \Phi(x, y)}{\partial \tau(x)} \varphi(y) d s(y), \quad x \in \partial D,
\end{array}
$$

where

$$
\Phi(x, y):=\frac{i}{4} H_{0}^{(1)}(k|x-y|), \quad x, y \in \mathbb{R}^{2}, \quad x \neq y,
$$

denotes the fundamental solution of the Helmholtz equation in $\mathbb{R}^{2}$ in terms of the Hankel function $H_{0}^{(1)}$ of the first kind and order zero. In fact, let $\partial D \in \mathcal{C}^{2, \alpha}$, then the operator $K^{\prime}: L^{2}(\partial D) \rightarrow L^{2}(\partial D)$ is compact, the operator $H^{\prime}: L^{2}(\partial D) \rightarrow L^{2}(\partial D)$ is bounded.

The corresponding inverse problem consists in determining the shape of the scatterer from the knowledge of the far-field pattern, i.e., in solving the ill-posed and nonlinear equation

$$
F(\partial D)=u_{\infty}
$$


for the unknown boundary $\partial D$ where $F$ denotes the operator which for the fixed incident field $u^{i}$ maps the boundary curve $\partial D$ onto the far-field pattern $u_{\infty}$ of the scattered wave $u^{s}$. We parameterize the boundary $\partial D$ as

$$
\gamma(t):=q(t)(\cos t, \sin t), \quad t \in[0,2 \pi],
$$

where $q(t)$ is a $2 \pi$-periodic real function. For simplicity of calculation, we assume that $q(t)$ to have the form of a trigonometric polynomial of degree $J$, in particular,

$$
q(t)=\sum_{j=0}^{J} \alpha_{j} \cos (j t)+\sum_{j=1}^{J} \beta_{j} \sin (j t), \quad \alpha_{j}, \beta_{j} \in \mathbb{R} .
$$

Definition 2.1. Let $X$ be normed space, $Y$ be Banach space, $U \subset X$ be an open set, if there exists an element $r_{0} \in U$, a bounded linear operator $\frac{\partial F}{\partial r} \in \mathcal{L}(X, Y)$ and a neighborhood of $r_{0}$ in $X$, such that

$$
F\left(r_{0}+h\right)=F\left(r_{0}\right)+\frac{\partial F}{\partial r} h+o(\|h\|), \quad h \in V,
$$

then the operator $F: U \rightarrow Y$ is differentiable.

We can see that Fréchet derivative is a extension of general derivative. To make computing efficient, we combine the original density function and the term which only depends on the shape of the boundary to a new density function. Denote

$$
\psi(t):=\varphi(x(t))\left|x^{\prime}(t)\right|,
$$

by this kind of variable substitution, the Fréchet derivative of $\left|x^{\prime}(t)\right|$, which respect to the boundary doesn't need to be computed.

Define $F(\partial D)=F_{\gamma}$, where $\gamma$ is a regular parametrization of $\partial D$. Throughout this paper, we consider the linearized form

$$
F_{\gamma}^{\prime} h+F_{\gamma}=u_{\infty}
$$

of the nonlinear equation (2.6) to apply Newton method, where $h$ is the iterative update value of the unknown boundary.

The solution of the system (2.5) can be expressed in the form of single-layer potential

$$
u^{s}(x)=\int_{\partial D} \Phi(x, y) \varphi(y) d s(y), \quad x \in \mathbb{R}^{2} \backslash \bar{D},
$$

where $\varphi \in L^{2}(\partial D)$ is the unknown density. Using the jump relation of single-layer potential and the boundary condition of (2.5), we obtain

$$
-\varphi(x)+K^{\prime}[\varphi](x)+i \lambda H^{\prime}[\varphi](x)=2 f(x), \quad x \in \partial D .
$$


By the parametric representation of the boundary, Eq. (2.10) can be transformed into

$$
\begin{aligned}
& -\varphi(\gamma(t))\left|\gamma^{\prime}(t)\right|+2 \int_{0}^{2 \pi} \frac{\partial \Phi(\gamma(t), \gamma(\sigma))}{\partial v(\gamma(t))} \varphi(\gamma(\sigma))\left|\gamma^{\prime}(t)\right|\left|\gamma^{\prime}(\sigma)\right| d \sigma \\
& \quad+i \lambda \cdot 2 \int_{0}^{2 \pi} \frac{\partial \Phi(\gamma(t), \gamma(\sigma))}{\partial \tau(\gamma(t))} \varphi(\gamma(\sigma))\left|\gamma^{\prime}(t)\right|\left|\gamma^{\prime}(\sigma)\right| d \sigma \\
& =2 f(\gamma(t))\left|\gamma^{\prime}(t)\right|,
\end{aligned}
$$

for $t \in[0,2 \pi]$. Denote $\psi(t):=\varphi(\gamma(t))\left|\gamma^{\prime}(t)\right|, g(t):=2 f(\gamma(t))\left|\gamma^{\prime}(t)\right|$, then we obtain

$$
\begin{array}{ll}
\tilde{K}[\psi](t)=2 \int_{0}^{2 \pi} \frac{\partial \Phi(\gamma(t), \gamma(\sigma))}{\partial v(\gamma(t))} \psi(\sigma)\left|\gamma^{\prime}(t)\right| d \sigma, & t \in[0,2 \pi], \\
\tilde{H}[\psi](t)=2 \int_{0}^{2 \pi} \frac{\partial \Phi(\gamma(t), \gamma(\sigma))}{\partial \tau(\gamma(t))} \psi(\sigma)\left|\gamma^{\prime}(t)\right| d \sigma, & t \in[0,2 \pi] .
\end{array}
$$

Consequently, Eq. (2.11) can be written as

$$
(-I+\tilde{K}+i \lambda \tilde{H})[\psi](t)=g(t) .
$$

The inverse operator of $-I+\tilde{K}+i \lambda \tilde{H}$ exists [25], so we have

$$
\psi(t)=(-I+\tilde{K}+i \lambda \tilde{H})^{-1} g(t) .
$$

Our inverse problem is given the measurable far-field data $u_{\infty}$ to solve the nonlinear illposed equation (2.6) by Newton method. Now based on the above discussion, an initial boundary is given, we get $F_{\gamma}$ from the direct problem, then compute $h$ from the linear integral equation (2.8), the updated boundary $\tilde{\gamma}=\gamma+h$ is obtained. At last the updated boundary is substituted into Eq. (2.11) as the initial boundary to continue the iteration.

\section{Fréchet differentiability of the far-field operator}

In order to use Newton-type method to solve this inverse scattering problem we have to study Fréchet differentiability of the far-field operator with respect to the parametrization $\gamma$ of the boundary $\partial D$. For this we first study the differentiability of the scattered wave $u^{s}$ with respect to $\gamma$. Later we obtain from the far-field representation the Fréchet differentiability of $F$. In the following analysis we denote by $C^{k}[0,2 \pi]$ the space of $2 \pi$ periodic k-times continuously differentiable functions over $[0,2 \pi]$.

By single-layer potential, scattering wave can be expressed as below

$$
u^{s}(x)=\int_{\partial D} \Phi(x, y) \varphi(y) d s(y), \quad x \in \mathbb{R}^{2} \backslash D, \quad y \in \partial D,
$$

the parameter form can be written as

$$
S(\psi ; \gamma)(x):=u^{S}(x)=\int_{0}^{2 \pi} \Phi(x, \gamma(\sigma)) \psi(\sigma) d \sigma, \quad x \in \mathbb{R}^{2} \backslash D .
$$


We compute the Fréchet derivative of the operator $(-I+\tilde{K}+i \lambda \tilde{H})^{-1}$ respect to the boundary $\gamma[19]$

$$
\begin{aligned}
{\left[(-I+\tilde{K}+i \lambda \tilde{H})^{-1}\right]_{\gamma}^{\prime} } & =-(-I+\tilde{K}+i \lambda \tilde{H})^{-1}(-I+\tilde{K}+i \lambda \tilde{H})_{\gamma}^{\prime}(-I+\tilde{K}+i \lambda \tilde{H})^{-1} \\
& =-(-I+\tilde{K}+i \lambda \tilde{H})^{-1}\left([\tilde{K}]_{\gamma}^{\prime}+i \lambda[\tilde{H}]_{\gamma}^{\prime}\right)(-I+\tilde{K}+i \lambda \tilde{H})^{-1}
\end{aligned}
$$

We denote $\tilde{S}_{\gamma}^{\prime}(\psi ; \gamma)[h]$ as the Fréchet derivative of $S(\psi ; \gamma)$ respect to the boundary $\gamma$. Using the chain rule of Fréchet derivative, we have

$$
\begin{aligned}
\tilde{S}_{\gamma}^{\prime}(\psi ; \gamma)[h]=\{ & \left.S\left((-I+\tilde{K}+i \lambda \tilde{H})^{-1}[\gamma] \cdot g[\gamma] ; \gamma\right)\right\}_{\gamma}^{\prime} \\
=S_{\gamma}^{\prime} & \left((-I+\tilde{K}+i \lambda \tilde{H})^{-1}[\gamma] \cdot g[\gamma] ; \gamma\right)[h] \\
& +S\left(-(-I+\tilde{K}+i \lambda \tilde{H})^{-1}\left([\tilde{K}]_{\gamma}^{\prime}+i \lambda[\tilde{H}]_{\gamma}^{\prime}\right)(-I+\tilde{K}+i \lambda \tilde{H})^{-1} \cdot g[\gamma] ; \gamma\right)[h] \\
& +S\left(-(-I+\tilde{K}+i \lambda \tilde{H})^{-1} \cdot g_{\gamma}^{\prime} ; \gamma\right)[h] \\
= & S_{\gamma}^{\prime}(\psi ; \gamma)[h]+S\left(-(-I+\tilde{K}+i \lambda \tilde{H})^{-1}\left([\tilde{K}]_{\gamma}^{\prime}+i \lambda[\tilde{H}]_{\gamma}^{\prime}\right)(\psi ; \gamma)[h]\right. \\
& \left.+(-I+\tilde{K}+i \lambda \tilde{H})^{-1} \cdot g_{\gamma}^{\prime}(\gamma)[h]\right) .
\end{aligned}
$$

Theorem 3.1. The integral operators $S, \tilde{K}$ and $\tilde{H}$ are Fréchet differentiable. The Fréchet derivatives are obtained by differentiation of their kernels, respectively [21].

From Definition 2.1 and Theorem 3.1, we get the scattered wave $u^{s}$ is differentiable with respect to $\gamma$. Then we are able to obtain the main result of this section.

Theorem 3.2. The far-field operator $F: \gamma \rightarrow u_{\infty}$ is Fréchet differentiable from $C^{2}[0,2 \pi]$ into $L^{2}(\Omega)$.

Proof. Let $\tilde{D}$ be a domain of $\mathbb{R}^{2}$ with the property $\bar{D} \cap \bar{D}=\varnothing$. By applying Fréchet differentiability of the scattered wave $u^{s}$ we get the existence of a constant $C$ with

$$
\left\|S(\psi ; r+h)-S(\psi ; r)-\tilde{S}_{\gamma}^{\prime}(\psi ; \gamma)[h]\right\|_{C^{2}(\tilde{D})} \leq C\|h\|_{C^{2}[0,2 \pi]}^{2}
$$

for sufficiently small $h$. From Green's representation theorem we have

$$
u_{\infty}(\hat{x})=\frac{1}{4 \pi} \int_{\partial D}\left\{u^{s}(y) \frac{\partial u^{i}(y,-\hat{x})}{\partial v(y)}-u^{i}(y,-\hat{x}) \frac{\partial u^{s}(y)}{\partial v}\right\} d s(y), \quad \hat{x} \in \Omega .
$$

From this, using the Cauchy-Schwarz inequality we derive that

$$
\left\|u_{\infty}(\psi ; r+h)-u_{\infty}(\psi ; r)-\tilde{u}_{\gamma, \infty}^{\prime}(\psi ; \gamma)[h]\right\|_{L^{2}(\Omega)} \leq C|| h \|_{C^{2}[0,2 \pi]}^{2}
$$

for all sufficiently small $h$. 


\section{The iterative method of the inverse problem}

In this section, under the frame of Newton method, we establish a numerical method for shape reconstruction of the scatterer. The problem of shape reconstruction is using the measurable far-field data $u_{\infty}$ to solve a nonlinear ill-posed equation $F(\partial D)=u_{\infty}$ with respect to the unknown boundary $\partial D$. First, the linearized form $F_{\gamma}^{\prime} h+F_{\gamma}=u_{\infty}$ of the nonlinear equation is obtained by using the Fréchet derivatives, where $h$ is the iterative update value of the unknown boundary, $\gamma$ is the parameter of the boundary. Then Newton method is used to solve the problem. Given an initial boundary $\gamma$, the corresponding density function is obtained by solving the boundary condition equation. The far-field model $F_{\gamma}$ and the Fréchet derivatives of far-field, which are related to the boundary can be calculated. The inverse problem is ill-posed, we use Tikhonov regularization to solve the equation and use Projection method to solve the inverse problem. After solving the above linear problem, we get the boundary update value $h$, finally we obtain the new boundary $\tilde{\gamma}=\gamma+h$. Continue iterating until stop criteria is met.

To get $F_{\gamma}^{\prime} h$ is actually to compute the far-field data of $\tilde{S}_{\gamma}^{\prime}(\psi ; \gamma)[h]$. Our result is stated as follows.

Theorem 4.1. The Fréchet derivative of the far-field operator is given by

$$
F_{\gamma}^{\prime} h=\sqrt{\frac{k}{8 \pi}} e^{-i \pi / 4} \int_{0}^{2 \pi} e^{-i k \hat{x}(t) \cdot \gamma(\sigma)}\langle\hat{x}(t), h(\sigma)\rangle \psi(\sigma) d \sigma+\frac{e^{i \pi / 4}}{\sqrt{8 k \pi}} \int_{0}^{2 \pi} e^{-i k \hat{x}(t) \cdot \gamma(\sigma)} \Psi(\sigma) d \sigma,
$$

where $\psi$ is a solution of the integral equation (2.12), and $\Psi$ has the following representation

$$
-(-I+\tilde{K}+i \lambda \tilde{H})^{-1}\left([\tilde{K}]_{\gamma}^{\prime}+i \lambda[\tilde{H}]_{\gamma}^{\prime}\right)(\psi ; \gamma)[h]+(-I+\tilde{K}+i \lambda \tilde{H})^{-1} \cdot g_{\gamma}^{\prime}(\gamma)[h] .
$$

Proof. Due to the chain rule of Fréchet derivative we discussed above,

$$
\begin{gathered}
\tilde{S}_{\gamma}^{\prime}(\psi ; \gamma)[h]=S_{\gamma}^{\prime}(\psi ; \gamma)[h]+S\left(-(-I+\tilde{K}+i \lambda \tilde{H})^{-1}\left([\tilde{K}]_{\gamma}^{\prime}+i \lambda[\tilde{H}]_{\gamma}^{\prime}\right)(\psi ; \gamma)[h]\right. \\
\left.+(-I+\tilde{K}+i \lambda \tilde{H})^{-1} \cdot g_{\gamma}^{\prime}(\gamma)[h]\right) .
\end{gathered}
$$

Denote by

$$
\begin{aligned}
V_{\gamma}^{\prime}(\psi ; \gamma)[h]:=S & -(-I+\tilde{K}+i \lambda \tilde{H})^{-1}\left([\tilde{K}]_{\gamma}^{\prime}+i \lambda[\tilde{H}]_{\gamma}^{\prime}\right)(\psi ; \gamma)[h] \\
& \left.+(-I+\tilde{K}+i \lambda \tilde{H})^{-1} \cdot g_{\gamma}^{\prime}(\gamma)[h]\right),
\end{aligned}
$$

then $\tilde{S}_{\gamma}^{\prime}(\psi ; \gamma)[h]$ can be written as

$$
\tilde{S}_{\gamma}^{\prime}(\psi ; \gamma)[h]=S_{\gamma}^{\prime}(\psi ; \gamma)[h]+V_{\gamma}^{\prime}(\psi ; \gamma)[h] .
$$

By using (3.1),we get

$$
S_{\gamma}^{\prime}(\psi ; \gamma)[h]=\int_{0}^{2 \pi}-\langle\operatorname{grad} \Phi(x, \gamma(\sigma)), h(\sigma)\rangle \psi(\sigma) d \sigma .
$$


Based on the relationship between scattering wave and far field, we have

$$
S_{\gamma, \infty}^{\prime}(\psi ; \gamma)[h](\hat{x})=\sqrt{\frac{k}{8 \pi}} e^{-i \pi / 4} \int_{0}^{2 \pi} e^{-i k \hat{x}(t) \cdot \gamma(\sigma)}\langle\hat{x}(t), h(\sigma)\rangle \psi(\sigma) d \sigma .
$$

Now we deal with $V_{\gamma}^{\prime}(\psi ; \gamma)[h]$, we find that

$$
V_{\gamma}^{\prime}(\psi ; \gamma)[h]=S(\Psi)
$$

which yields to

$$
V_{\gamma, \infty}^{\prime}(\psi ; \gamma)[h]=S_{\infty}(\Psi)=\frac{e^{i \pi / 4}}{\sqrt{8 k \pi}} \int_{0}^{2 \pi} e^{-i k \hat{x}(t) \cdot \gamma(\sigma)} \Psi(\sigma) d \sigma .
$$

We can conclude

$$
\begin{aligned}
F_{\gamma}^{\prime} h= & \tilde{S}_{\gamma, \infty}^{\prime}(\psi ; \gamma)[h]=S_{\gamma, \infty}^{\prime}(\psi ; \gamma)[h]+V_{\gamma, \infty}^{\prime}(\psi ; \gamma)[h] \\
= & \sqrt{\frac{k}{8 \pi} e^{-i \pi / 4} \int_{0}^{2 \pi} e^{-i k \hat{x}(t) \cdot \gamma(\sigma)}\langle\hat{x}(t), h(\sigma)\rangle \psi(\sigma) d \sigma} \\
& +\frac{e^{i \pi / 4}}{\sqrt{8 k \pi}} \int_{0}^{2 \pi} e^{-i k \hat{x}(t) \cdot \gamma(\sigma)} \Psi(\sigma) d \sigma .
\end{aligned}
$$

Thus, we complete the proof.

By using

$$
\begin{aligned}
\tilde{K}[\psi, \gamma](t) & =2 \int_{0}^{2 \pi} \frac{\partial \Phi(\gamma(t), \gamma(\sigma))}{\partial v(\gamma(t))} \psi(\sigma)\left|\gamma^{\prime}(t)\right| d \sigma \\
& =2 \int_{0}^{2 \pi}\langle\operatorname{grad} \Phi(\gamma(t), \gamma(\sigma)), n(\gamma(t))\rangle \psi(\sigma) d \sigma
\end{aligned}
$$

where $\langle x, y\rangle=x \cdot y$ denotes the inner product of $x$ and $y$,

$$
n(\gamma(t))=\left|\gamma^{\prime}(t)\right| v(\gamma(t))=\left(\gamma_{2}^{\prime}(t),-\gamma_{1}^{\prime}(t)\right),
$$

we derive the Fréchet derivative of the operator $\tilde{K}$ as below $[19,21]$,

$$
\begin{aligned}
{[\tilde{K}]_{\gamma}^{\prime}(\psi ; \gamma)[h](t)=} & 2 \int_{0}^{2 \pi}\langle\operatorname{grad}\langle\operatorname{grad} \Phi(\gamma(t), \gamma(\sigma)), h(t)-h(\sigma)\rangle, n(\gamma(t))\rangle \psi(\sigma) d \sigma \\
& \quad+2 \int_{0}^{2 \pi}\langle\operatorname{grad} \Phi(\gamma(t), \gamma(\sigma)), n(h(t))\rangle \psi(\sigma) d \sigma \\
= & \int_{0}^{2 \pi} Q(t, \sigma) \psi(\sigma) d \sigma
\end{aligned}
$$


where

$$
\begin{aligned}
Q(t, \sigma)=- & \frac{i k}{2} H_{1}^{(1)}(k|\gamma(t)-\gamma(\sigma)|) \frac{\langle\gamma(t)-\gamma(\sigma), n(h(t))\rangle}{|\gamma(t)-\gamma(\sigma)|} \\
& -\frac{i k^{2}}{2} H_{0}^{(1)}(k|\gamma(t)-\gamma(\sigma)|) \frac{\langle\gamma(t)-\gamma(\sigma), h(t)-h(\sigma)\rangle}{|\gamma(t)-\gamma(\sigma)|^{2}}(\langle\gamma(t)-\gamma(\sigma), n(\gamma(t))\rangle) \\
& +i k H_{1}^{(1)}(k|\gamma(t)-\gamma(\sigma)|) \frac{\langle\gamma(t)-\gamma(\sigma), h(t)-h(\sigma)\rangle}{|\gamma(t)-\gamma(\sigma)|^{2}} \frac{\langle\gamma(t)-\gamma(\sigma), n(\gamma(t))\rangle}{|\gamma(t)-\gamma(\sigma)|} \\
& -\frac{i k}{2} H_{1}^{(1)}(k|\gamma(t)-\gamma(\sigma)|) \frac{\langle h(t)-h(\sigma), n(\gamma(t))\rangle}{|\gamma(t)-\gamma(\sigma)|}
\end{aligned}
$$

The singular kernel decomposition of $Q(t, \sigma)$ can be established as follows

(1) for $t \neq \sigma$, we get

$$
\begin{aligned}
& Q_{1}(t, \sigma)=- \frac{1}{2 \pi}\left\{-k J_{1}(k|\gamma(t)-\gamma(\sigma)|) \frac{\langle\gamma(t)-\gamma(\sigma), n(h(t))\rangle}{|\gamma(t)-\gamma(\sigma)|}\right. \\
&-k^{2} J_{0}(k|\gamma(t)-\gamma(\sigma)|) \frac{\langle\gamma(t)-\gamma(\sigma), h(t)-h(\sigma)\rangle}{|\gamma(t)-\gamma(\sigma)|^{2}}(\langle\gamma(t)-\gamma(\sigma), n(\gamma(t))\rangle) \\
&+2 k J_{1}(k|\gamma(t)-\gamma(\sigma)|) \frac{\langle\gamma(t)-\gamma(\sigma), h(t)-h(\sigma)\rangle}{|\gamma(t)-\gamma(\sigma)|^{2}} \frac{\langle\gamma(t)-\gamma(\sigma), n(\gamma(t))\rangle}{|\gamma(t)-\gamma(\sigma)|} \\
&\left.-k J_{1}(k|\gamma(t)-\gamma(\sigma)|) \frac{\langle h(t)-h(\sigma), n(\gamma(t))\rangle}{|\gamma(t)-\gamma(\sigma)|}\right\}, \\
& Q_{2}(t, \sigma)=Q(t, \sigma)-Q_{1}(t, \sigma) \ln \left(4 \sin ^{2} \frac{t-\sigma}{2}\right) .
\end{aligned}
$$

(2) for $t=\sigma$, we have

$$
\begin{aligned}
& Q_{1}(t, t)=0, \\
& Q_{2}(t, t)=\frac{1}{2 \pi}\left[\frac{\left\langle\gamma^{\prime \prime}(t), n(h(t))\right\rangle+\left\langle h^{\prime \prime}(t), n(\gamma(t))\right\rangle}{\left|\gamma^{\prime}(t)\right|^{2}}-2 \frac{\left\langle\gamma^{\prime \prime}(t), n(\gamma(t))\right\rangle\left\langle\gamma^{\prime}(t), h^{\prime}(t)\right\rangle}{\left|\gamma^{\prime}(t)\right|^{4}}\right] .
\end{aligned}
$$

Based on

$$
\begin{aligned}
\tilde{H}[\psi, \gamma](t) & =2 \int_{0}^{2 \pi} \frac{\partial \Phi(\gamma(t), \gamma(\sigma))}{\partial \tau(\gamma(t))} \psi(\sigma)\left|\gamma^{\prime}(t)\right| d \sigma \\
& =2 \int_{0}^{2 \pi}\langle\operatorname{grad} \Phi(\gamma(t), \gamma(\sigma)), p(\gamma(t))\rangle \psi(\sigma) d \sigma
\end{aligned}
$$

where $\langle x, y\rangle=x \cdot y$ denotes the inner product of $x$ and $y$,

$$
p(\gamma(t))=\left|\gamma^{\prime}(t)\right| \tau(\gamma(t))=\left(\gamma_{1}^{\prime}(t), \gamma_{2}^{\prime}(t)\right),
$$


the Fréchet derivative of the operator $\tilde{H}$ is given by

$$
\begin{aligned}
{[\tilde{H}]_{\gamma}^{\prime}(\psi ; \gamma)[h](t)=} & 2 \int_{0}^{2 \pi}\langle\operatorname{grad}\langle\operatorname{grad} \Phi(\gamma(t), \gamma(\sigma)), h(t)-h(\sigma)\rangle, p(\gamma(t))\rangle \psi(\sigma) d \sigma \\
& +2 \int_{0}^{2 \pi}\langle\operatorname{grad} \Phi(\gamma(t), \gamma(\sigma)), p(h(t))\rangle \psi(\sigma) d \sigma \\
= & \int_{0}^{2 \pi} G(t, \sigma) \psi(\sigma) d \sigma
\end{aligned}
$$

where

$$
\begin{aligned}
G(t, \sigma)=- & \frac{i k}{2} H_{1}^{(1)}(k|\gamma(t)-\gamma(\sigma)|) \frac{\langle\gamma(t)-\gamma(\sigma), p(h(t))\rangle}{|\gamma(t)-\gamma(\sigma)|} \\
& -\frac{i k^{2}}{2} H_{0}^{(1)}(k|\gamma(t)-\gamma(\sigma)|) \frac{\langle\gamma(t)-\gamma(\sigma), h(t)-h(\sigma)\rangle}{|\gamma(t)-\gamma(\sigma)|^{2}}(\langle\gamma(t)-\gamma(\sigma), p(\gamma(t))\rangle) \\
& +i k H_{1}^{(1)}(k|\gamma(t)-\gamma(\sigma)|) \frac{\langle\gamma(t)-\gamma(\sigma), h(t)-h(\sigma)\rangle}{|\gamma(t)-\gamma(\sigma)|^{2}} \frac{\langle\gamma(t)-\gamma(\sigma), p(\gamma(t))\rangle}{|\gamma(t)-\gamma(\sigma)|} \\
& -\frac{i k}{2} H_{1}^{(1)}(k|\gamma(t)-\gamma(\sigma)|) \frac{\langle h(t)-h(\sigma), p(\gamma(t))\rangle}{|\gamma(t)-\gamma(\sigma)|} .
\end{aligned}
$$

The singular kernel decomposition of $G(t, \sigma)$ can be shown as follows

(1) for $t \neq \sigma$, we get

$$
\begin{aligned}
& G_{1}(t, \sigma)=- \frac{1}{2 \pi}\left\{-k J_{1}(k|\gamma(t)-\gamma(\sigma)|) \frac{\langle\gamma(t)-\gamma(\sigma), p(h(t))\rangle}{|\gamma(t)-\gamma(\sigma)|}\right. \\
&-k^{2} J_{0}(k|\gamma(t)-\gamma(\sigma)|) \frac{\langle\gamma(t)-\gamma(\sigma), h(t)-h(\sigma)\rangle}{|\gamma(t)-\gamma(\sigma)|^{2}}(\langle\gamma(t)-\gamma(\sigma), p(\gamma(t))\rangle) \\
&+2 k J_{1}(k|\gamma(t)-\gamma(\sigma)|) \frac{\langle\gamma(t)-\gamma(\sigma), h(t)-h(\sigma)\rangle}{|\gamma(t)-\gamma(\sigma)|^{2}} \frac{\langle\gamma(t)-\gamma(\sigma), p(\gamma(t))\rangle}{|\gamma(t)-\gamma(\sigma)|} \\
&\left.-k J_{1}(k|\gamma(t)-\gamma(\sigma)|) \frac{\langle h(t)-h(\sigma), p(\gamma(t))\rangle}{|\gamma(t)-\gamma(\sigma)|}\right\}, \\
& G_{2}(t, \sigma)=G(t, \sigma)-G_{1}(t, \sigma) \ln \left(4 \sin ^{2} \frac{t-\sigma}{2}\right)-\frac{1}{2 \pi} \cot \frac{\sigma-t}{2} .
\end{aligned}
$$

(2) for $t=\sigma$, we have

$$
\begin{aligned}
& G_{1}(t, t)=0, \\
& G_{2}(t, t)=-\frac{1}{2 \pi}\left[\frac{\left\langle\gamma^{\prime \prime}(t), p(h(t))\right\rangle+\left\langle h^{\prime \prime}(t), p(\gamma(t))\right\rangle}{\left|\gamma^{\prime}(t)\right|^{2}}-2 \frac{\left\langle\gamma^{\prime \prime}(t), p(\gamma(t))\right\rangle\left\langle\gamma^{\prime}(t), h^{\prime}(t)\right\rangle}{\left|\gamma^{\prime}(t)\right|^{4}}\right] .
\end{aligned}
$$


When the incident wave $u^{i}=e^{i k \gamma \cdot d}$, we obtain

$$
\begin{aligned}
g(t) & =-2\left(\frac{\partial u^{i}}{\partial v(\gamma)}\left|\gamma^{\prime}(t)\right|+i \lambda \frac{\partial u^{i}}{\partial \tau(\gamma)}\left|\gamma^{\prime}(t)\right|\right) \\
& =-2\left(\frac{\partial e^{i k \gamma \cdot d}}{\partial v(\gamma)}\left|\gamma^{\prime}(t)\right|+i \lambda \frac{\partial e^{i k \gamma \cdot d}}{\partial \tau(\gamma)}\left|\gamma^{\prime}(t)\right|\right) \\
& =-2\left(\left\langle\operatorname{grad} e^{i k \gamma \cdot d}, v(\gamma(t))\right\rangle\left|\gamma^{\prime}(t)\right|+i \lambda\left\langle\operatorname{grad} e^{i k \gamma \cdot d}, \tau(\gamma(t))\right\rangle\left|\gamma^{\prime}(t)\right|\right) \\
& =-2\left(\left\langle\operatorname{grad} e^{i k \gamma \cdot d}, n(\gamma(t))\right\rangle+i \lambda\left\langle\operatorname{grad} e^{i k \gamma \cdot d}, p(\gamma(t))\right\rangle\right) \\
& =-2\left(g_{1}(t)+i \lambda g_{2}(t)\right) .
\end{aligned}
$$

Then the Fréchet derivative of $g_{1}(t)$ is given by

$$
\begin{aligned}
g_{1}^{\prime}(\gamma)[h](t) & =\left\langle\operatorname{grad} e^{i k \gamma \cdot d}, n(h(t))\right\rangle+\left\langle\operatorname{grad}\left\langle\operatorname{grad} e^{i k \gamma \cdot d}, h(t)\right\rangle, n(\gamma(t))\right\rangle \\
& =i k e^{i k \gamma \cdot d}\langle d, n(h(t))\rangle+\left\langle\operatorname{grad}\left\langle e^{i k \gamma \cdot d} i k d, h(t)\right\rangle, n(\gamma(t))\right\rangle \\
& =i k e^{i k \gamma \cdot d}\langle d, n(h(t))\rangle+\left\langle e^{i k \gamma \cdot d} i k d, h(t)\right\rangle\langle i k d, n(\gamma(t))\rangle \\
& =i k e^{i k \gamma \cdot d}\langle d, n(h(t))\rangle-k^{2} e^{i k \gamma \cdot d}\langle d, h(t)\rangle\langle d, n(\gamma(t))\rangle,
\end{aligned}
$$

while the Fréchet derivative of $g_{2}(t)$ can be established as

$$
g_{2}^{\prime}(\gamma)[h](t)=i k e^{i k \gamma \cdot d}\langle d, p(h(t))\rangle-k^{2} e^{i k \gamma \cdot d}\langle d, h(t)\rangle\langle d, p(\gamma(t))\rangle
$$

Thus, we derive the Fréchet derivative of $g(t)$

$$
g_{\gamma}^{\prime}(\gamma)[h](t)=-2\left(g_{1}^{\prime}(\gamma)[h](t)+i \lambda g_{2}^{\prime}(\gamma)[h](t)\right) .
$$

We note both the update value $h$ and the boundary are $2 \pi$-period functions, so we can use trigonometric interpolation polynomials to approximate $h$, i.e.,

$$
h(t)=\sum_{l=0}^{2 m} \chi_{l} p_{l}(t),
$$

where $\chi_{l}$ is an unknown coefficient, $p_{l}(t)=B(l t)(\cos (t), \sin (t))$, with

$$
B(l t)= \begin{cases}\cos (l t), & l=0, \cdots, m, \\ \sin ((l-m) t), & l=m+1, \cdots, 2 m .\end{cases}
$$

By the linearity of the operator $F_{\gamma}^{\prime}$, Eq. (2.8) can be rewritten as

$$
\sum_{l=0}^{2 m} \chi_{l} F_{\gamma}^{\prime}\left(p_{l}(t)\right)+F_{\gamma}(\hat{x}(t))=u_{\infty}(\hat{x}(t)) .
$$


From

$$
\begin{aligned}
& S_{\gamma, \infty}^{\prime}(\psi ; \gamma)[h](\hat{x})=\sum_{l=0}^{2 m} \chi_{l} S_{\gamma, \infty}^{\prime}(\psi ; \gamma)\left[p_{l}\right](\hat{x}), \\
& V_{\gamma, \infty}^{\prime}(\psi ; \gamma)[h](\hat{x})=\sum_{l=0}^{2 m} \chi_{l} V_{\gamma, \infty}^{\prime}(\psi ; \gamma)\left[p_{l}\right](\hat{x}),
\end{aligned}
$$

with

$$
\begin{aligned}
& S_{\gamma, \infty}^{\prime}(\psi ; \gamma)\left[p_{l}\right](\hat{x})= \sqrt{\frac{k}{8 \pi}} e^{-i \pi / 4} \int_{0}^{2 \pi} e^{-i k \hat{x}(t) \cdot \gamma(\sigma)}\left\langle\hat{x}(t), p_{l}(\sigma)\right\rangle \psi(\sigma) d \sigma \\
& V_{\gamma, \infty}^{\prime}(\psi ; \gamma)\left[p_{l}\right]=S_{\infty}\left((-I+\tilde{K}+i \lambda \tilde{H})^{-1}\left([\tilde{K}]_{\gamma}^{\prime}+i \lambda[\tilde{H}]_{\gamma}^{\prime}\right)(\psi ; \gamma)\left[p_{l}\right]\right. \\
&\left.+(-I+\tilde{K}+i \lambda \tilde{H})^{-1}(g)_{\gamma}^{\prime}(\gamma)\left[p_{l}\right]\right)
\end{aligned}
$$

we obtain

$$
\sum_{l=0}^{2 m} \chi_{l} F_{\gamma}^{\prime}\left(p_{l}(t)\right)=\sum_{l=0}^{2 m} \chi_{l} S_{\gamma, \infty}^{\prime}(\psi ; \gamma)\left[p_{l}\right](t)+\sum_{l=0}^{2 m} \chi_{l} V_{\gamma, \infty}^{\prime}(\psi ; \gamma)\left[p_{l}\right](t) .
$$

Therefor, based on (4.2a) and (4.2b) we can get $S_{\gamma, \infty}^{\prime}(\psi ; \gamma)\left[p_{l}\right]$ and $V_{\gamma, \infty}^{\prime}(\psi ; \gamma)\left[p_{l}\right]$, then $F_{\gamma}^{\prime}\left(p_{l}\right)$ can be computed by (4.3).

Given $u_{\infty}, F_{\gamma}, F_{\gamma}^{\prime}\left(p_{l}(t)\right)$, we get $\chi_{l}$ by solving Eq. (4.1). However, because the equation is ill-posed, Tikhonov regularization is needed. We transform solving Eq. (4.1) into minimizing the functional

$$
W:=\sum_{j=0}^{2 n-1}\left|\sum_{l=0}^{2 m} \chi_{l} F_{\gamma}^{\prime}\left(p_{l}\left(t_{j}\right)\right)+F_{\gamma}\left(\hat{x}_{j}\right)-u_{\infty}\left(\hat{x}_{j}\right)\right|^{2}+\alpha \sum_{l=0}^{2 m}\left|\chi_{l}\right|^{2},
$$

where $\alpha$ is the regularization parameter. That is, to solve the unknown coefficient $\chi_{l}$, such that (4.4) is minimized.

\section{Numerical examples of inverse problem}

In this section, some numerical examples are given to verify the feasibility of the Newton method we proposed above. In the numerical examples, we get the unknown coefficient $\chi_{l}$ from (4.4) by Newton method.

This method can be summarized as four steps:

Step 1 Given the initial boundary $\gamma$, after obtaining the density function $\psi$, compute the far-field model $F_{\gamma}$ which corresponding to the initial boundary.

Step 2 Compute $S_{\gamma, \infty}^{\prime}(\psi ; \gamma)\left[p_{l}\right], V_{\gamma, \infty}^{\prime}(\psi ; \gamma)\left[p_{l}\right]$. We note the density function $\psi$ is the same as in Step 1, so plug $\psi$ into (4.3) to obtain $F_{\gamma}^{\prime}\left(p_{l}\right)$. 
Step 3 By using the regularization method, the unknown coefficient $\chi_{l}$ is solved according to $u_{\infty}$ and $F_{\gamma}, F_{\gamma}^{\prime}$, which are calculated in the first two steps. Then, it is substituted into $h=\sum_{l=0}^{2 m} \chi_{l} p_{l}$ to get the incremental $h$ of the boundary $\gamma$, and the updated boundary $\tilde{\gamma}=\gamma+h$ is obtained.

Step 4 Repeat the above steps until the stop iteration criterion is satisfied.

Using the far-field data of an incident wave to reconstruct the closed region, the direction is $d=(1,0)$, the wave number is $k=1$, the number of configuration points is $n=32$. The regularization parameters are selected by the general deviation principle in [10]. The error data is given by the following formula

$$
u_{\infty}^{\delta}:=u_{\infty}+\delta \frac{\left\|u_{\infty}\right\|_{L^{2}}}{\|\eta\|_{L^{2}}} \eta
$$

where $\eta$ is normal distribution variable, $\delta$ is the relative noise level. In all the examples, We choose the circle $(\cos (t), \sin (t))$ as the initial value of the boundary, $\lambda=0.05$ as the impedance coefficient. All the numerical examples show the results of 10 steps of iteration.

Example 5.1. We consider the parametric form of pear-shaped boundary,

$$
\partial D=\{x \mid x(t)=(1.2+0.25 \cos (t))(\cos (t), \sin (t)), t \in[0,2 \pi]\} .
$$

The expansion number of the boundary increment $h$ is $m=3$. Using the exact data $u_{\infty}$ and the noisy data with $\delta=0.05,10$ steps iteration, the reconstruction of the pear-shaped are established in Fig. 1.

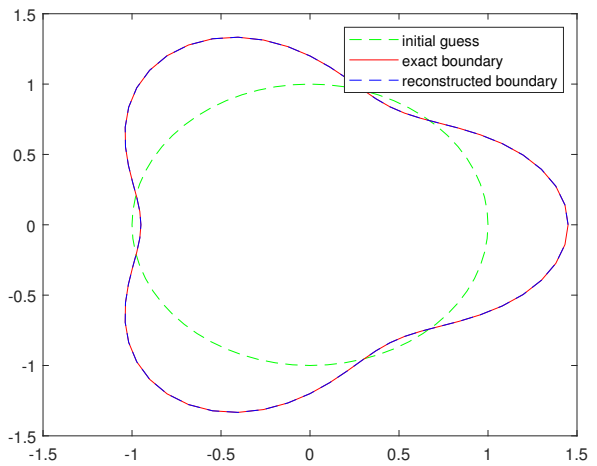

(a)

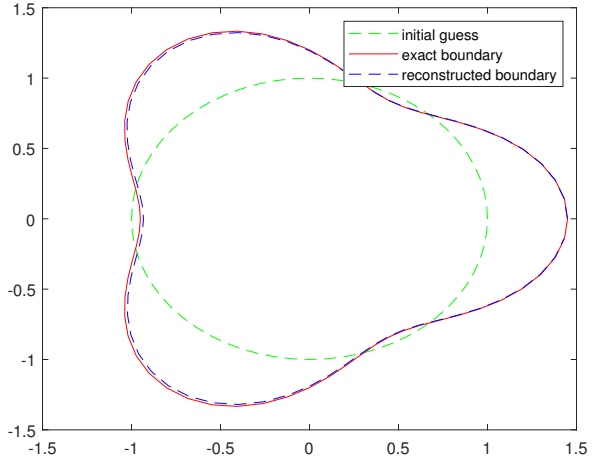

(b)

Figure 1: Reconstruction of pear-shaped with exact data on the left and with $5 \%$ noisy data on the right. 
Example 5.2. We consider the parametric form of heart-shaped boundary,

$$
\partial D=\left\{x \mid x(t)=\left(1.2-\frac{1}{3} \sin (t)-\frac{1}{7} \sin (3 t)\right)(\cos (t), \sin (t)), t \in[0,2 \pi]\right\} .
$$

The expansion number of the boundary increment $h$ is $m=2$. Using the exact data $u_{\infty}$ and the noisy data with $\delta=0.05,10$ steps iteration, the reconstruction of the heart-shaped are established in Fig. 2.

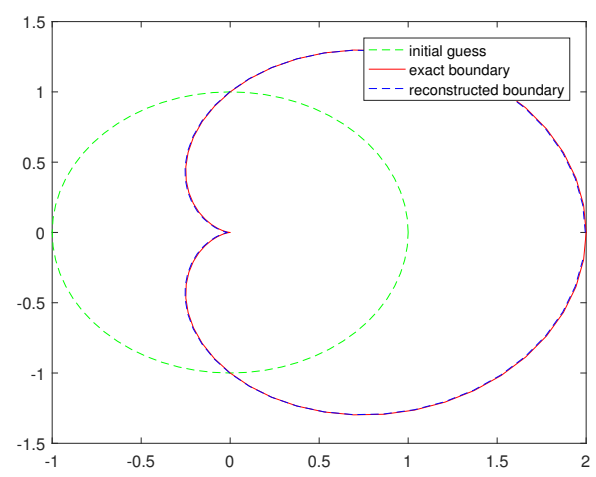

(a)

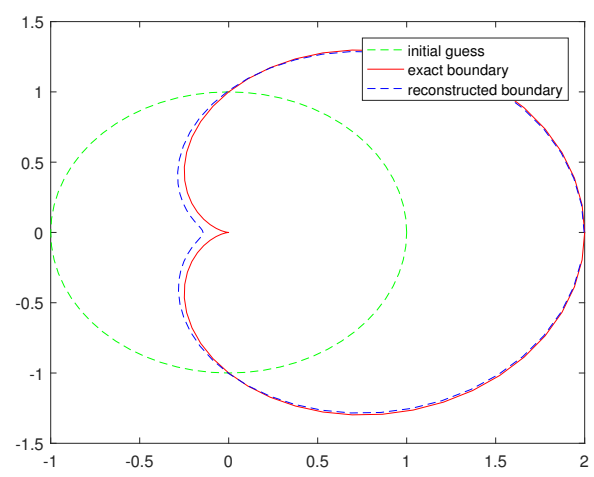

(b)

Figure 2: Reconstruction of heart-shaped with exact data on the left and with $5 \%$ noisy data on the right.

Example 5.3. We consider the parametric form of peanut-shaped boundary,

$$
\partial D=\left\{x \mid x(t)=0.8 \sqrt{\cos ^{2}(t)+0.25 \sin ^{2}(t)}(\cos (t), \sin (t)), t \in[0,2 \pi]\right\} .
$$

The expansion number of the boundary increment $h$ is $m=3$. Using the exact data $u_{\infty}$ and the noisy data with $\delta=0.05,10$ steps iteration, the reconstruction of the peanut-shaped are established in Fig. 3.

Reconstructions of pear and heart are better than peanut, mainly because the parametric representation of the peanut-shaped boundary cannot be written as $q(t)$ in (2.7).

\section{Acknowledgements}

The research of $\mathrm{Y}$. Q. Hu is supported by $\mathrm{PhD}$ research startup foundation of Jinling Institute of Technology (No. jit-b-201524) and the Science Foundation of Jinling Institute of Technology (No. Jit-fhxm-201809). The author would like to thank the referees for the helpful suggestions. 


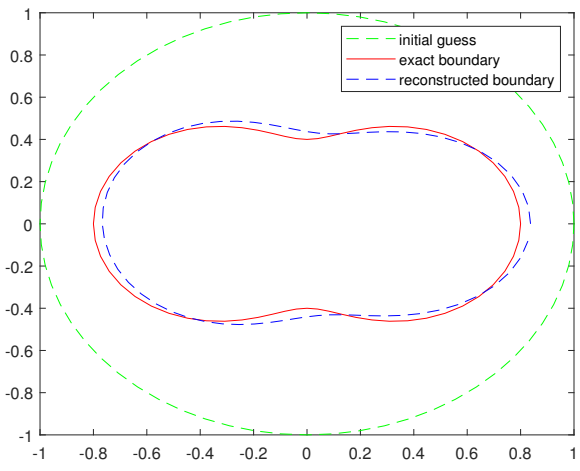

(a)

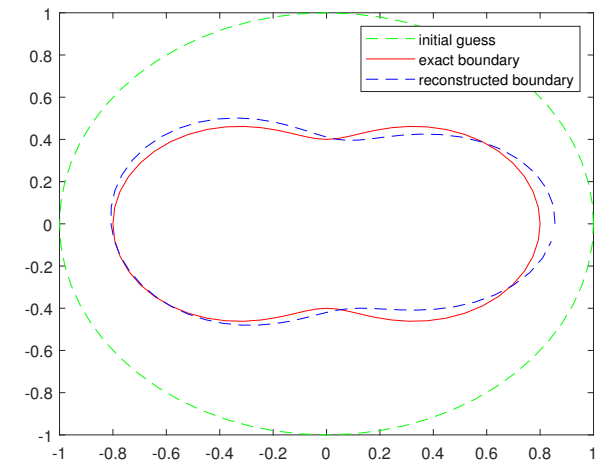

(b)

Figure 3: Reconstruction of peanut-shaped with exact data on the left and with 5\% noisy data on the right.

\section{References}

[1] F. CAKOnI AND D. COLTON, Qualitative Methods in Inverse Scattering Theory, SpringerVerlag, Berlin, 2006.

[2] F. CAKONI, Y. Q. HU AND R. KRESS, Simultaneous reconstruction of shape and generalized impedance functions in electrostatic imaging, Inverse Problems, 30(10) (2014), pp. 105009.

[3] F. CAKONI, O. IVANYSHYN, R. KRESS, AND F. LE LOUER, A boundary integral equations for the transmission eigenvalue problem for Maxwell equations, Math. Methods Appl. Sci., 41(4) (2018), pp. 1316-1330.

[4] F. CAKONI AND R. KRESS, Integral equations for inverse problems in corrosion detection from partial Cauchy data, Inverse Problems Imag., 1(2) (2007), pp. 229-245.

[5] F. CAKONI AND R. KRESS, A boundary integral equation method for the transmission eigenvalue problem, Appl. Anal., 96 (2017), pp. 23-38.

[6] F. CAKONI, R. KRESS AND C. SCHUFT, Integral equations for shape and impedance reconstruction in corrosion detection, Inverse Problems, 26(9) (2010), pp. 095012.

[7] F. CAKONI, R. KRESS AND C. SCHUFT, Simultaneous reconstruction of shape and impedance in corrosion detection, Methods Appl. Anal., 17(4) (2010), pp. 331-462.

[8] D. COlTON AND R. KRess, Integral Equation Methods in Scattering Theroy, New York: John Wiley \& Sons, 1983.

[9] D. COlTON AND R. KRESS, Inverse Acoustic and Electromagnetic Scattering Theory, Applied Mathematical Sciences, Springer, New York, 2019.

[10] P. C. HANSEN, Regularization Tools: A Matlab package for analysis and solution of discrete illposed problems, Numer. Algorithms, 46 (2007), pp. 189-194.

[11] Y. KIAN, D. SAMBOU AND E. SOCCORSI, Logarithmic stability inequality in an inverse source problem for the heat equation on a waveguide, Appl. Anal., 99 (2020), pp. 2210-2228.

[12] R. KRESS, Linear Integral Equations, Springer-Verlag, New York, 1999.

[13] R. KRESS AND W. RUNDELL, Nonlinear integral equations and the iterative solution for an inverse boundary value problem, Inverse Problems, 21 (2005), pp. 1207-1223.

[14] P. A. KRUTITSKII, On the electric current from electrodes in a magnetized semiconductor film, IMA 
J. Appl. Math., 60 (1998), pp. 285-297.

[15] P. A. KRUTITSKII AND A. O. CHIKILEV, The method of integral equations in the oblique derivatives problem arising in semiconductor physics, Differential Equations, 36 (2000), pp. 1323-1336.

[16] P. A. KRUTITSKII, The oblique derivative problem for the Helmholtz equation and scattering tidal waves, Prec. Royal Soc. A, 457 (2001), pp. 1735-1755.

[17] J. LI AND G. SUN, A nonlinear integral equation method for the inverse scattering problem by sound-soft rough surfaces, Inverse Problems Sci. Eng., 23(4) (2015), pp. 557-577.

[18] P. A. MARTiN, On the diffractioon of Poincaré waves, Math. Methods Appl. Sci., 24 (2001), pp. 913-925.

[19] L. MÖNCH, A Newton method for solving the inverse scattering problem for a sound-hard obstacle, Inverse Problems, 12 (1996), pp. 309-323.

[20] A. MUNNIER AND K. RAMDANI, Calderón cavities inverse problem as a shape-from-moments problem, Quart. Appl. Math., 76 (2018), pp. 407-435.

[21] R. POTTHAST, Fréchet differentiability of boundary integral operators in inverse acoustic scattering, Inverse Problems, 10 (1994), pp. 431-447.

[22] H. H. QIN AND F. CAKONI, Nonlinear integral equations for shape reconstruction in the inverse interior scattering problem, Inverse Problems, 27(3) (2011), pp. 035005.

[23] H. B. WANG AND J. J. LIU, The two-dimensional direct and inverse scattering problems with generalized oblique derivative boundary condition, SIAM J. Appl. Math., 75 (2015), pp. 313-334.

[24] H. B. WANG AND J. J. LIU, An inverse scattering problem with generalized oblique derivative boundary condition, Appl. Numer. Math., 108 (2016), pp. 226-241.

[25] H. B. WANG AND G. NAKAMURA, The integral equation method for electromagnetic scattering problem at oblique incidence, Appl. Numer. Math., 62 (2012), pp. 860-873. 